Financial Statistical Journal (2018) Volume 1 doi:10.24294/fsj.v1i3.609

\title{
The Impact of Socioeconomic Status on the Psychological Distress of Breast Cancer Patients in Kolkata"
}

Arunima Datta $^{1^{*}}$, Rimi Sharma ${ }^{2}$

${ }^{1}$ Dept. of Psychology, NetajiSubhas Chandra Bose Cancer Research Institute, 16A, Park Lane, Kolkata-700016, Kolkata, West Bengal, India. Email Address: arunima.datta8@gmail.com

Contact No.:7890924589

${ }^{2}$ Dept. of Psychology, Neotia University, Kolkata, West Bengal, India.

\begin{abstract}
Background: Breast cancer patients experience a variety of psychological symptoms such as anxiety, depression that affect their quality of life. The present study probed whether the socioeconomic status had any significance on psychological distress and quality of life of breast cancer patients in India.

Method: This was a cross-sectional and observational study, conducted over 111 breast cancer patients in a tertiary cancer hospital, Kolkata. Subjects were eligible to participate and they were of over 23rd years of age and had a histologically confirmed diagnosis of breast cancer had no history of recurrence or metastasis, and had no previous psychological problems. Age, marital status, residence, education, occupation and family income were assesses for patients' socio economic status. Psychological symptoms and quality of life were assessed by using validated tools.Means and standard deviations of each outcome were compared by socioeconomic status and multivariate linear regression models for evaluating the association between socioeconomic status, psychological distress and quality of life
\end{abstract}

Results: The patient group was categorized into three groups based on their income level as follows: $\leq 500=27.9 \%$ ), $500-1000=51.4 \%$ and high income $=\geq 1000=20.7 \%$. A total of 111 breast cancer patients participated in the study; the mean age of participants was 49.7 ( $\mathrm{SE}=1.31$ ).After using multivariate logistic regression with fully adjusted models, the patients who were living alone had significantly higher level of depression and poor quality of life compared to those who are employed. Higher education and family income both positively associated with quality of life after adjusting for age, marital status and occupation.

Conclusion: The findings revealed that the family income have a significant impact on the psychological distress of the cancer patients.

Keywords: Breast Cancer; Psychological Factors; Socioeconomic

\section{Introduction}

Generally in India, there is limited number of investigations done about the impact of socioeconomic status and its impact on the breast cancer patients. Although many studies have been conducted on cancer patients in general, the number of studies done on breast cancer patients, specifically, is limited. Following are some of the studies conducted in order to understand the link between SES and psychological distress among cancer patients: This study is to understand the impact of socioeconomic status on the Psychological distress of cancer and cardiovascular disease patients in Kerala region of India. This study was conducted on a total of 225 women patients belonging to Kerala, in the year 2016 by Alex. The findings of this study were that individuals belonging to a low socioeconomic background were more concerned about the expenses that were to be born for their treatment. They were also more worried about managing their day-to-day expenses. Thus, the study revealed that the socioeconomic status does play an important role in the Psychological distress of an individual with cancer and cardiovascular disease experiences.

Oliver Chang et al., in 2014 had worked over association between socioeconomic status and altered appearance

Copyright (C) 2018 Arunima Datta et al.

doi: $10.24294 /$ fsj.v1i3.609

EnPress Publisher LLC.This work is licensed under the Creative Commons Attribution-NonCommercial 4.0 International License (CC BY-NC 4.0). http://creativecommons.org/licenses/ by/4.0/ 
distress, body image and quality of life among breast cancer patients. Through their study they had said that socioeconomic status is significantly associated with altered appearance distress, body image, and quality of life in Korean women with breast cancer. Patients who suffer from altered appearance distress or lower body image are much more likely to experience psychosocial, physical, and functional problems than women who do not, therefore health care providers should be aware of the changes and distresses that these breast cancer patients go through and provide specific information and psychosocial support to socioeconomically more vulnerable patients.

Socioeconomic determinants of health have been discussed in the literature, and the need to tailor patient care has been widely expressed (Preston, 2010). Studies regarding socioeconomic status and its differing effects on the health of breast cancer survivors have focused primarily on assessing clinical and pathologic characteristics, quality of life, depression, and anxiety (King et al., 2000; Karakoyun-Celik et al., 2010; SchmidBuchi et al., 2013; Wang et al., 2013)

In the year 2009, Limin et al conducted a study on the impact of socioeconomic status on cancer incidence and stage at diagnosis: selected findings from the surveillance, epidemiology and end results. Through their study they con-cluded that individuals (men and women) whose educational levels were lower than high school degrees, and also those individuals who had an annual income lower than $\$ 12500$, had higher rates of cancer incidence as compared to the individuals who had acquired college degrees, and also from the group of people having annual income of more than $\$ 50,000$. They also concluded that lower income was significantly a factor for the increased chances of distant-stage breast cancer in people.

Psychological well-being and the quality of life he or she has, influenced to a large extent by the impact of socio economic status. It is known that low socioeconomic status is linked with more occurrences of stressful events and the lack of coping methods (Eron et al 1982). It is seen that poor coping skills and the life events that the individual faces are correlated with psychological well-being and their quality of life. People belonging to a higher socioeconomic status have been seen to have lower chances of acquiring and suffering from most psychiatric disorders and medical conditions. They have been known to have lower mortality rates from these conditions, which also includes cancer (Adler et al., 1994).

We have followed their study to investigate the impact of SES on breast cancer patients of a different populationthe eastern region population of West Bengal. How this psychological states as well as socioeconomic status interfere in a patient's quality of life was also discussed.

\section{Materials and Methods \\ Design:}

This was observational study was designed using validated tools and structured face to face interview schedule.

\section{Patient Selection:}

For the present study participants were recruited between July 1 and October 30, 2017, from NetajiSubhas Chandra Bose Cancer Research Institute, Kolkata.

The researchers had approached 168 breast cancer survivors; $131(72.02 \%)$ agreed to participate in the study. Among them, women who had previous history of psychological problem $(n=3)$, recurrence of the disease ( $n=6)$, or who were stage $4(n=11)$ were excluded from the study, resulting in a total of 111 study participants.

To evaluate the impact of socioeconomic status on psychological problems, we used the data of the only 111 diagnosed with breast cancer within less than 12 months. Their consent was also taken. Demographic information was taken from the patients including, their age, marital status, residence, and income. On the basis of their per capita income, patients were divided into three groups: below 500, 500 to 1000 and above 1000 . All patients were made to go through the assessment of depression, anxiety and quality of life. Inclusion and exclusion criteria are given below.

\section{Inclusion Criteria}

Patients diagnosed with breast cancer;

Permanent resident of India

People belonging to initial stage, or adverse stage of cancer; 


\section{Exclusion}

People with any other forms of cancer, patients with previous history of mental disorders or illness; newly diagnosed breast cancer patients.

\section{Data collection}

The structured interview was conducted using proper performa for taking demographical and clinoco-pathological details. After that depression, anxiety and quality of life were assessed by using validated tools.

\section{STAI-II: State-Trait Anxiety Inventory}

The Bengali version of State-Trait Anxiety Inventory was used on the patients. It is a 40-item self-report questionnaire. Every item is rated by the patient on a 4-point rating scale. The state version of the inventory measures a temporary anxiety state of the patients, whereas the trait version of the inventory measures a long-term and more stable anxiety experience of the patients. (Eskelinen $\mathrm{M}$ et al., 2011)

Beck Depression Inventory (BDI):- This is a 21 item questionnaire consisting of six sub-scales, Mild mood disturbance, Borderline clinical depression, Moderate depression, severe depression and Extreme depression. Each item is rated in a 3 point scale. (Eskelinen M et al., 2011)

EORTC: European Organization for Research and Treatment of Cancer breast cancer-specific quality-of-life questionnaire module

EORTC QLQ-BR23 is a questionnaire used to measure the quality of life in cancer patients. It is a self-administered questionnaire administered on breast cancer patients who are at various stages of treatment. There are 5 domains that the questionnaire administers: sexuality, body image, breast symptoms, arm symptoms, and systemic therapy side effects. (May Leng Tan et al. 2014)

\section{Procedure}

After maintaining exclusion and inclusion criteria, patients were selected for the present study. Ethical concern was approved following ICMR rule. Demographic information were taken from both groups (including, age, marital status, residence, occupation, family type and family income). After taking data, use proper statistics for making conclusion.

\section{Statistical Analysis}

We scored anxiety, depression and quality of life according to the manual of the Beck Depression Inventory, State trait Anxiety Inventory and EORTC QLQ-BR23 respectively. For assessing the impact of socio economic status on depression, anxiety and quality of life, we calculated the mean and standard error of the each response and compared them with socio economic status. To analyze the relation between socio economic status and anxiety, depression and quality of life we used multivariate linear regression model. In the multivariate analysis, we adjusted for variables that could be potential variables: age, residence, relationship status, education, occupation and family income.All statistical analyses were performed using SPSS program version 21. Statistical significance was defined as $\mathrm{p}<0.05$.

\section{Results}

Table 1 showed the distribution of sociodemographic factors among breast cancer patients. According to age group, it showed that $9 \%$ patients belonged to age group $<30$ years; $31.5 \%$ belonged to $30-40$ years; $21 \%$ belonged to age-group of 40-50 years; $23 \%$ belonged to age-group of 50-60 years and $22 \%$ patients were above the age of 60 .

In the marital status bar graph, we can see that $77.5 \%$ people were living with their spouses, and $22.5 \%$ people were living alone. The chart of education level indicates that $44.1 \%$ individuals had primary level of education, $33.3 \%$ people had completed high school degrees and $22.5 \%$ people were graduate degree holders.

Out of the total, $49.5 \%$ lived in rural areas, whereas $50.5 \%$ lived in urban areas. Through this, we found that out of the total breast cancer patients, $20.7 \%$ people belonged to income group 3 that is whose monthly income was more than 10000 INR. 51.4\% people belonged to income group 2, whose monthly income was between $5000-10000$ and $27.9 \%$ people belonged to income group 1, with income below 5000 INR.

Breast cancer patients with less education suffered from higher level of psychological distress than those with 
higher education- depression: (36.3 vs 34.22); anxiety: (39.02 vs 41.25); quality of life: (17.31 vs 18.36) . In terms of monthly family income, patients who had higher income $(>1000)$ reported better psychologically stable than patients with lower income (<500)- depression: (41.71 vs 37.36); anxiety : (53.8 vs 45.33); quality of life: (13.71 vs 21.21$)$, and it was statistically significant. Although major psychological symptoms as well as quality of life seemed to be affected by a patient's age, relationship status and her residence.

Table 2 shows the distribution of clinicopathological factors of breast cancer patients. The menopausal status of the breast cancer patients shows that in pre-menopausal state, $44.1 \%$ women were present in total, and $55.9 \%$ patients were there in post-menopausal state.

\begin{tabular}{|c|c|c|c|c|}
\hline $\begin{array}{l}\text { Sociodemographic } \\
\text { factor }\end{array}$ & No of cases & $\begin{array}{l}\text { BDI } \\
(\mathrm{Mean} \pm \mathrm{SE})\end{array}$ & $\begin{array}{l}\text { STAI } \\
(\mathrm{Mean}+\mathrm{SE})\end{array}$ & $\begin{array}{l}\text { EORTC QLQ-BR23 } \\
(\text { Mean } \pm \text { SE) }\end{array}$ \\
\hline Age & s & 2 & 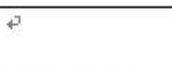 & \\
\hline$\leq 30$ years & $10(9.0 \%)$ & $35.25 \pm 9.28$ & $50.13 \pm 6.61$ & $17.46 \pm 1.28$ \\
\hline $30-40$ & $35(31.5 \%)$ & $28.47 \pm 2.48$ & $49.46 \pm 3.1$ & $17.77 \pm 1.12$ \\
\hline $40-50$ & $21(18.9 \%)$ & $30.11 \pm 2.51$ & $49.43 \pm 3.06$ & $15.05 \pm 0.96$ \\
\hline $50-60$ & $23(20.7 \%)$ & $28.31 \pm 1.89$ & $49.14 \pm 3.65$ & $16.5 \pm 1.09$ \\
\hline$>60$ years & $22(19.8 \%)$ & $35 \pm 0.12$ & $44.5 \pm 2.01$ & $17 \pm 0.13$ \\
\hline MaritalStatus & 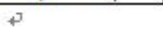 & 3 & 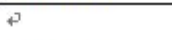 & \\
\hline Living with spouse & $86(77.5 \%)$ & $28.61 \pm 2.19$ & $48 \pm 2.22$ & $16.93 \pm 0.62$ \\
\hline Living without spouse & $25(22.5 \%)$ & $30.60 \pm 1.64$ & $49.74 \pm 2.65$ & $18.76 \pm 1.35$ \\
\hline Residence & a & 4 & 4 & 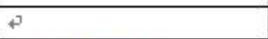 \\
\hline Rural & $55(49.5 \%)$ & $27.8 \pm 1.63$ & $49.04 \pm 2.81$ & $17.33 \pm 0.86$ \\
\hline urban & $56(50.5 \%)$ & $31.37 \pm 2.27$ & $49.72 \pm 2.62$ & $17.36 \pm 0.78$ \\
\hline Education & p & 4 & s & \\
\hline Primary School & $49(44.1 \%)$ & $36.3 \pm 1.21$ & $39.02 \pm 0.08$ & $17.31 \pm 0.86$ \\
\hline Higher Secondary & $37(33.3 \%)$ & $35.6 \pm 1.3$ & $41.13 \pm 2.1$ & $16.67 \pm 1.01$ \\
\hline Graduate & $25(22.5 \%)$ & $34.22 \pm 2.4$ & $41.25 \pm 0.32$ & $18.36 \pm 1.2$ \\
\hline $\begin{array}{l}\text { Precipitate Family } \\
\text { income }\end{array}$ & 8 & 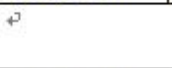 & 3 & \\
\hline$<500{ }^{\circ}$ & $31(27.9 \%)$ & $41.71 \pm 4.70$ & $53.8 \pm 2.77$ & $13.71 \pm 1.11$ \\
\hline $500-1000$ & $57(51.4 \%)$ & $39.55 \pm 1.40$ & $54.33 \pm 2.88$ & $20.85+0.84$ \\
\hline$>1000$ & $23(20.7 \%)$ & $37.36 \pm 2.59$ & $45.33 \pm 1.83$ & $21.21+1.31$ \\
\hline
\end{tabular}

Table 1. Socio demographic characteristics of Participants $(\mathrm{N}=111)$

\begin{tabular}{|c|c|c|c|c|}
\hline Clinical Characteristic & No. of Cases & $\begin{array}{l}\text { BDI } \\
(\text { Mean } \pm \text { SE) }\end{array}$ & $\begin{array}{l}\text { STAI } \\
(\text { Mean } \pm \text { SE) }\end{array}$ & $\begin{array}{l}\text { EORTC QLQ-BR23 } \\
(\text { Mean } \pm \text { SE) }\end{array}$ \\
\hline \multicolumn{5}{|l|}{ Menopausal Status } \\
\hline Pre & $49(44.1 \%)$ & $33.64 \pm 5.85$ & $52.09 \pm 1.93$ & $19.32 \pm 0.96$ \\
\hline Post & $62(55.9 \%)$ & $32.1 \pm 3.2$ & $51.5 \pm 3.61$ & $20.63 \pm 0.77$ \\
\hline \multicolumn{5}{|l|}{ Histology } \\
\hline Ductal & $90(81.1 \%)$ & $31.5 \pm 2.1$ & $46.14 \pm 1.89$ & $20.41 \pm 0.71$ \\
\hline Lobular & $21(18.9 \%)$ & $30.4 \pm 0.65$ & $46.1 \pm 1.13$ & $19.1 \pm 1.18$ \\
\hline \multicolumn{5}{|l|}{ Estrogen Receptor } \\
\hline Positive & $77(69.4 \%)$ & $34.28 \pm 2.31$ & $49.12 \pm 1.79$ & $20.23 \pm 0.72$ \\
\hline Negative & $34(30.6 \%)$ & $37.42 \pm 2.74$ & $46.71 \pm 3.15$ & $19.64 \pm 1.15$ \\
\hline \multicolumn{5}{|l|}{ Progesteron Receptor } \\
\hline Positive & $73(65.8 \%)$ & $32.21 \pm 2.17$ & $50.67 \pm 2.98$ & $20.23 \pm 1.16$ \\
\hline Negative & $38(34.2 \%)$ & $33.63 \pm 2.89$ & $48.24 \pm 2.23$ & $19.70 \pm 1$ \\
\hline \multicolumn{5}{|l|}{ Her2/neu status } \\
\hline Over express & $72(64.9 \%)$ & $33.81 \pm 2.11$ & $47.64 \pm 2.73$ & $20.33 \pm 0.74$ \\
\hline Not over express & $39(35.1 \%)$ & $30 \pm 2.52$ & $50.8 \pm 2.46$ & $19.52 \pm 1.07$ \\
\hline \multicolumn{5}{|l|}{ Number of chemotherapy } \\
\hline$\leq 6^{\text {th }}$ cycle & $79(71.2 \%)$ & $31.82 \pm 1.59$ & $47.52 \pm 2.1$ & $19.99 \pm 0.76$ \\
\hline More than $6^{\text {th }}$ & $32(28.8 \%)$ & $28 \pm 1.5$ & $43.78 \pm 3.95$ & $20.22 \pm 0.98$ \\
\hline \multicolumn{5}{|l|}{ Treatment Plan } \\
\hline Neo-adjuvant chemotherapy & $68(61.3 \%)$ & $33.62 \pm 3.02$ & $49.19 \pm 3.89$ & $21.1 \pm 1.11$ \\
\hline Adjuvant chemotherapy & $43(38.7 \%)$ & $32.19 \pm 1.96$ & $47.78 \pm 2.05$ & $20.3 \pm 0.78$ \\
\hline
\end{tabular}

Table 2. ClinicopathocopathologicalFactors of Participants $(\mathrm{N}=111)$ 
In the histology bar graph, it can be seen that $81.9 \%$ patients had ductal and $18.9 \%$ patients had lobular problems. The estrogen receptor bar graph displays that $69.4 \%$ patients had positive and $30.6 \%$ patients had negative feedbacks. In progesterone bar graph we see that a total $65.8 \%$ patients had positive and $34.2 \%$ patients had negative feedback. The Her $2 /$ neu status bar graph showed that over expression occurred in $64.9 \%$ patients and non-over expression was seen in $35.1 \%$ patients.

When considering the number of chemotherapy, it was seen that $71.2 \%$ patients had 6 or less numbers of chemotherapy and $28.8 \%$ patients had taken more than 6 chemotherapy. In the treatment plan graph, neo-adjuvent chemotherapy was done for $61.3 \%$ patients and $38.7 \%$ patients had got adjuvant chemotherapy.

When considering number of chemotherapy, it can be seen that patients with 6 or less chemotherapy had higher depression score than those with more than 6 chemotherapy (31.82 vs 28). Patients with 6 or below chemotherapy also displayed higher anxiety score than those with more than 6 chemotherapy (47.52 vs 43.78). The score on quality of life was also lower in cases of patients receiving 6 or less numbers of chemotherapy than those with more than 6 chemotherapy (19.9 vs 20.22).

When considering the treatment plan, it was observed that the depression levels was higher in neo-adjuvant chemotherapy than in adjuvant chemotherapy patients (33.62 vs 32.19). The score of anxiety was also marked higher in neo-adjuvant chemotherapy patients than in adjuvant chemotherapy patients (49.19 vs 47.78). The score of quality of life of patients with neo-adjuvant chemotherapy treatment was surprisingly higher than that of adjuvant chemotherapy treatment patients (21.1 vs 20.3).

Factors associated with psychological symptoms including anxiety and depression and quality of life The results of multivariate analysis of factors associated with psychological symptoms and quality of life are displayed in Table 3. With the adjustment for demographic factors including age, relationship status, residence, education and socioeconomic status as well as clinicopathological factors such as menopausal status, current treatment status, and number of chemotherapy was significantly associated with altered appearance distress, body image, and quality of life. The patients who had higher level of family income $(>1000)$ were more likely to have better psychological management $\left(\mathrm{OR}=1,95 \% \mathrm{CI}(2.83-3.06)^{*}\right.$ than patients who had lower income $(<500)$. In case of quality of life, patients living without spouse had poorer quality of life $((\mathrm{OR}=1.5,95 \%$ CI (5.83-7.02) than patients living with a spouse $(p<0.05)$.Patients with graduate level of education had better quality of life by 7.16 points than patients with primary level of education after adjusting for all other factors, and it was statistically significant $(\mathrm{p}<0.01)$.

\section{Discussion}

Oliver Chang et al., in 2014 had worked over association between socioeconomic status and altered appearance distress, body image and quality of life among breast cancer patients. Through their study they had said that socioeconomic status is significantly associated with altered appearance distress, body image, and quality of life in Korean women with breast cancer. Patients who suffer from altered appearance distress or lower body image are much more likely to experience psychosocial, physical, and functional problems than women who do not.

Booth et al presented their study impact of socioeconomic status on Stage of Cancer at Diagnosis Survival in the year 2010. The study was conducted in Ontario, Canada. They studied the impact of SES on cancer patients of Canada. They investigated that even though the government provided the residents with overall world class healthcare services, the residents' SES did play an important role in the survival rates of the patients. This study focused on forms of cancer, such as, breast, colon, NSCL, cervical and laryngeal cancers.

Following above mentioned studies we want to describe the impact of socioeconomic characteristics on psychological problems and quality of life among 111 breast cancer patients who experienced anxiety and depression during the period of cancer treatment in West Bengal. We found that a patient's educational level and family income were positively associated with psychological problems. As a result it affects their quality of life.

In West Bengal, most of the women after marriage occupationally are housewives. They are more depending on their husband economically rather than emotionally. In such situation when they had diagnosed as a breast cancer 
patient and when they see their family members devote everything for getting well soon but they are helpless. As a result their response was "I want to live for my family members not for that I want to live. I want to make them successful. But, unfortunately I cannot... due to day to day deterioration of health". So, in this situation socioeconomic status laid an import role for developing psychological distress and that affect their quality of life. Support with numerous previous findings in our study, quality of life increased for patients who were married and for patients with more education than patients who were living alone or patients with less education (Janz et al., 2005; Ahn et al., 2009; Ashing-Giwa and Lim, 2009; Salonen et al., 2009).

Education of the practical situation as well as for cancer is very important for leading a better quality of this result had contradicted with Ashing-Giwa and Lim, 2009. They had found that physicians spend more time with affluent and educated patients than with financially and educationally deprived patients who actually need more attention and care. For people belonging to low socioeconomic statuses affording the expenses of cancer care for a prolonged period of time can prove to be a costly affair. Depending on their limited income for not only providing for daily expenditures, but also for an extra cost of medications, treatments and hospital visits can be an independent factor in development of psychological symptoms such as anxiety or depression. Also, for the breast cancer patients for whom the expenses are to be made may also feel responsible, and hence, helpless. The result of the present study was supported with Oliver Chang et al. 2014.The results of our present study indicate that socioeconomic factors could be better in dependent factors of a breast cancer patient's development of psychological problems and quality of life than clinical characteristics.

Most cancers are related to socioeconomic status with low SES individuals more at risk. Moreover, even incidence rates for major cancers have slowed or decreased, declines to morality have been slow in minority population than among whites (Glanz et al., 2003). One of the most potent risk factors for early disease disability and death is low socio-economic status (SES). From birth throughout life, those who are born into the lower social classes experience more and more intense stressors of all kinds, which have a cumulative toll on health risks. Lower income and poor educational and occupational attainment leads to exposure to a broad array of stressors including inadequate housing, violence, danger, lack of vital goods and services, inadequate medical facilities, poor sanitation, exposure to environmental pollutants and numerous other hazards. Leigh Ann Simmons et al., (2009) examined factors associated with self-reporting depression for low-income rural women experiencing depressive symptoms. Women reporting depression were significantly likelier to report physical health problems, injury/illness, and more frequent physician visits. Low socio-economic status is associated with a higher prevalence of depression, but it is not yet known whether change in socio-economic status leads to a change in rates of depression.

Vincent Lorant (2007) assessed whether longitudinal change in socio-economic factors affects change of depression level, socio-economic factors based on material standard of living, education, employment status and social relationships. A lowering in material standard of living between annual waves was associated with increase in depressive symptoms and caseness of major depression. Life circumstances also influenced depression. Ceasing to cohabit with a partner increased depressive symptoms and caseness, and improvement in circumstances reduced them; the negative effects were stronger than the positive ones. The study showed a clear relationship between worsening of socio-economic circumstances and depression.

\section{Conclusion}

Through the findings of this study, we aim to also acquire any kind of government funding for the patients belonging to low socioeconomic statuses for better possibilities and opportunities of their treatment. This will eliminate the chances of factors such as becoming a liability for their family, or dependency on family for bearing the costs of cancer treatment, to become a factor for developing psychological symptoms.

\section{Reference}

1. Adler, Boyce, N.E., Chesney, T., Cohen, M.A., Folkman, S., Kahn, S.(1994). Socioeconomic status and health The challenge of the gradient.The American Psychologist, 49(1), 15-24. 
2. Ahn E, Cho J, Shin DW, et al (2009). Impact of breast cancer diagnosis and treatment on work-related life and factors affecting them. Breast Cancer Res Treat, 116, 609-16.

3. Ashing-Giwa KT, Lim JW (2009). Examining the impact of socioeconomic status and socioecologic stress on physical and mental health quality of life among breast cancer survivors. OncolNurs Forum, 36, 79-88.

4. Ashing-Giwa KT, Lim JW (2009). Examining the impact of socioeconomic status and socioecologic stress on physical and mental health quality of life among breast cancer survivors. OncolNurs Forum, 36, 79-88.

5. Booth CM, Li G, Zhang-Salomons J, Mackillop WJ. (2010). Cancer. 1;116(17):4160-7. doi: 10.1002/cncr.25427.

6. Eron, Leonard D., Peterson, Rolf A. (1982). Abnormal behavior: Social approaches. Annual Review of Psychology, 33, 231-264.

7. Eskelinen M, et al. (2011). Assessment of general anxiety in patients with breast disease and breast cancer using the Spielberger STAI self evaluation test: a prospective case-control study in Finland. Anticancer Res. 2011 May;31(5):1801-6.

8. Janz NK, Mujahid M, Lantz PM, et al (2005). Population-based study of the relationship of treatment and sociodemographics on quality of life for early stage breast cancer. Qual Life Res, 14, 1467-79.

9. Karakoyun-Celik O, Gorken I, Sahin S, et al (2010). Depression and anxiety levels in woman under follow-up for breast cancer: relationship to coping with cancer and quality of life. Med Oncol, 27, 108-13.

10. Karen Glanz., Robert, T., Croyle., Veronica, Y., Chollette., \& Vivian, W. Pinn. (2003). Cancer-Related Health Disparities in Women.American Journal of Public Health, 93 (2), 292-298.

11. King MT, Kenny P, Shiell A, Hall J, Boyages J (2000). Quality of life three months and one year after first treatment for early stage breast cancer: influence of treatment and patient characteristics. Qual Life Res, 9, 789-800.

12. Lehman, Barbara J., Taylor, Shelley E., Kiefe, Catarina I., Seeman, Teresa E (2009). Relationship of early life stress and psychological functioning to blood pressure in the CARDIA study. HealthPsychology, 28(3), 338-346

13. Limin X. Clegg, Marsha E. Reichman, and Brenda K. Edwards. (2009).Impact of socioeconomic status on cancer incidence and stage at diagnosis: selected findings from the surveillance, epidemiology, and end results: National Longitudinal Mortality Study. Cancer Causes Control. 20(4): 10.1007/s10552-008-9256-0.

14. Lorant V, Demarest S, Miermans PJ, Van Oyen H. (2007). Survey error in measuring socio-economic risk factors of health status: a comparison of a survey and a census.36(6):1292-9.

15. May Leng Tan, DahlianaBinteIdris, Lee WahTeo, Soon YueLoh, GekChingSeow, Yen YenChia, AungSoe Tin. (2014). Validation of EORTC QLQ-C30 and QLQ-BR23 questionnaires in the measurement of quality of life of breast cancer patients in Singapore. Asia-Pacific Journal of Oncology Nursing. (1);1, 22-32.

16. Oliver Chang1\&, Eun-Kyung Choi\&, Im-Ryung Kim, Seok-Jin Nam, Jeong Eon Lee, Se Kyung Lee, Young-HyuckIm, YeonHee Park, Juhee Cho. (2014). Association between Socioeconomic Status and Altered Appearance Distress, Body Image, and Quality of Life Among Breast Cancer Patients. DOI:http://dx.doi.org/10.7314/APJCP.2014.15.20.8607.

17. RoshanAnie Alex (2016). The Impact of Socioeconomic Status on the Psychological Distress of Cancer and Cardiovascular Disease Patients. Imperial Journal of Interdisciplinary Research (IJIR). (2),4.

18. Salonen P, Tarkka MT, Kellokumpu-Lehtinen PL, et al (2009). Telephone intervention and quality of life in patients with breast cancer. Cancer Nurs, 32, 177-90.

19. Schmid-Buchi S, Halfens RJ, Muller M, Dassen T, van den Borne B (2013). Factors associated with supportive care needs of patients under treatment for breast cancer. Eur J OncolNurs, 17, 22-9.

20. Wang K, Li X, Zhou C, et al (2013). Socio-economic factors influencing tumor presentation and treatment options in Chinese breast cancer patients. Asian Pac J Cancer Prev, 14, 267-74. 